\title{
Scattering of Geometric Algebra Wave Functions and Collapse in Measurements
}

\author{
Alexander Soiguine \\ SOiGUINE Quantum Computing, Aliso Viejo, CA, USA \\ Email: alex@soiguine.com
}

How to cite this paper: Soiguine, A. (2020) Scattering of Geometric Algebra Wave Functions and Collapse in Measurements. Journal of Applied Mathematics and Physics, 8, 1838-1844.

https://doi.org/10.4236/jamp.2020.89138

Received: July 29, 2020

Accepted: September 15, 2020

Published: September 18, 2020

Copyright $\odot 2020$ by author(s) and Scientific Research Publishing Inc. This work is licensed under the Creative Commons Attribution International License (CC BY 4.0).

http://creativecommons.org/licenses/by/4.0/

\begin{abstract}
The research considers wavelike objects that are elements of even subalgebra of geometric algebra in three dimensions. The used formalism particularly eliminates long existing confusion about the reasons behind the appearance of the imaginary unit in quantum mechanics and introduces clear definition of wave functions. When a wave function acts through the Hopf fibration on a localized geometric algebra element, that is executing a measurement, the result can be named as "collapse" of the wave function.
\end{abstract}

\section{Keywords}

Wave Functions, Geometric Algebra, Measurements, Scattering, Entanglement, Dualism

\section{(c) (i) Open Access}

\section{Introduction}

The current research is based on long list of the author works on geometric algebra formalism in quantum mechanics [1] [2]. Previous results allow to implement the process of creating "particles" in "collapse" of wave function. The work aims at receiving explicit formulas identifying geometric objects appearing in interaction of wave functions with other objects.

The research considers wavelike objects that are elements of even subalgebra $G_{3}^{+}$of geometric algebra $G_{3}$ [1]. The used formalism particularly eliminates long existing confusion about the reasons behind the appearance of the imaginary unit $i$ in quantum mechanics [3], and introduces clear definition of wave functions.

The wave likeness follows from the fact that a $G_{3}^{+}$element scalar part and the coefficient of bivector part both can depend on time and three-dimensional position as: 


$$
R\left(\cos [\varphi(\omega t, \vec{r})]+I_{S} \sin [\varphi(\omega t, \vec{r})]\right) \equiv R \mathrm{e}^{I_{S} \varphi(\omega t, \vec{r})}
$$

Such elements are in the current research wave functions, also called in the case $R=1 \quad g$-qubits since they are generalization of commonly known quantum mechanical qubits when formal imaginary unit $i$ is replaced by a geometrically clear unit bivector $I_{S}$ in three dimensions.

The wave functions can be transformed, and scattered, for example, when another $G_{3}^{+}$element multiplies it (from the left.) The result of such time proportional actions is described in the geometric algebra terms by Schrodinger equation [4].

When a wave function acts through the Hopf fibration on a localized $G_{3}^{+}$ element, that's executing a measurement, the result can be named as "collapse" of the wave function though it is physical identity of different nature, output of Hopf fibration.

\section{Scattering of a Wave Function by $G_{3}^{+}$Element}

In the following, the wave functions will be taken of specific form:

$$
\begin{aligned}
& S p\left(\omega, \vec{r}, t, I_{S_{0}}, I_{B_{0}}, I_{E_{0}}\right) \\
& =2 \cos \left(\omega\left(\left[\left(I_{3} I_{S_{0}}\right) \cdot \vec{r}\right]\right)\right)\left(\cos (\omega t)+\frac{1}{\sqrt{2}} I_{S_{0}} \sin (\omega t)\right. \\
& \left.+\frac{1}{\sqrt{2}} I_{B_{0}} \cos (\omega t)+\frac{1}{\sqrt{2}} I_{E_{0}} \sin (\omega t)\right),
\end{aligned}
$$

where $I_{3}$ is unit value oriented 3-volume (here right hand screw oriented.)

I will call it sprefield, it is special solution of Maxwell equation calculated in geometric algebra terms [2]. The name follows from the fact that

$\operatorname{Sp}\left(\omega, \vec{r}, t, I_{S_{0}}, I_{B_{0}}, I_{E_{0}}\right)$ is non-local object instantly spreading its modifications through the whole $3 \mathrm{D}$ and time parameter values.

Sprefield when scattered on a $G_{3}^{+}$element

$$
\begin{aligned}
& \cos (\gamma)+\sin (\gamma)\left(\gamma_{1} I_{S_{0}}+\gamma_{2} I_{B_{0}}+\gamma_{3} I_{E_{0}}\right) \text { becomes }{ }^{1} \text { : } \\
& \left(\cos (\gamma)+\sin (\gamma)\left(\gamma_{1} I_{S_{0}}+\gamma_{2} I_{B_{0}}+\gamma_{3} I_{E_{0}}\right)\right) \operatorname{Sp}\left(\omega, \vec{r}, t, I_{S_{0}}, I_{B_{0}}, I_{E_{0}}\right) \\
& =2 \cos \left(\omega\left(\left[\left(I_{3} I_{S_{0}}\right) \cdot \vec{r}\right]\right)\right)\left[\frac { 1 } { \sqrt { 2 } } \left(\cos (\omega t) \cos (\gamma)-\gamma_{1} \sin (\gamma) \sin (\omega t)\right.\right. \\
& \left.-\gamma_{2} \sin (\gamma) \cos (\omega t)-\gamma_{3} \sin (\gamma) \sin (\omega t)\right)+\frac{1}{\sqrt{2}} I_{S_{0}}\left(\gamma_{1} \sin (\gamma) \cos (\omega t)\right. \\
& \left.+\cos (\gamma) \sin (\omega t)+\gamma_{3} \sin (\gamma) \cos (\omega t)-\gamma_{2} \sin (\gamma) \sin (\omega t)\right) \\
& +\frac{1}{\sqrt{2}} I_{B_{0}}\left(\gamma_{2} \sin (\gamma) \cos (\omega t)+\cos (\gamma) \cos (\omega t)+\gamma_{1} \sin (\gamma) \sin (\omega t)\right. \\
& \left.-\gamma_{3} \sin (\gamma) \sin (\omega t)\right)+\frac{1}{\sqrt{2}} I_{E_{0}}\left(\gamma_{3} \sin (\gamma) \cos (\omega t)+\cos (\gamma) \sin (\omega t)\right. \\
& \left.\left.+\gamma_{2} \sin (\gamma) \sin (\omega t)-\gamma_{1} \sin (\gamma) \cos (\omega t)\right)\right]
\end{aligned}
$$

${ }^{1}$ Multiplication of the sprefield by the scattering element from the left is taken for agreement with the left side multiplication by Hamiltonian in conventional quantum mechanics Schrodinger equation. 
This scattered sprefield is defined in all points $\vec{r}$ and time parameter values $t$, independently when scattering occurs.

That general case is not highly informative.

Consider special cases.

- Scattering element is $\cos (\gamma)+I_{S_{0}} \sin (\gamma)=\mathrm{e}^{I_{S_{0}} \gamma}$ with the result:

$$
\begin{aligned}
\left(\cos (\gamma)+I_{S_{0}} \sin (\gamma)\right) \operatorname{Sp}\left(\omega, \vec{r}, t, I_{S_{0}}, I_{B_{0}}, I_{E_{0}}\right) \\
=2 \cos \left(\omega\left(\left[\left(I_{3} I_{S_{0}}\right) \cdot \vec{r}\right]\right)\right)\left[\frac{1}{\sqrt{2}}(\cos (\omega t) \cos (\gamma)-\sin (\gamma) \sin (\omega t))\right. \\
+\frac{1}{\sqrt{2}} I_{S_{0}}(\sin (\gamma) \cos (\omega t)+\cos (\gamma) \sin (\omega t)) \\
+\frac{1}{\sqrt{2}} I_{B_{0}}(\cos (\gamma) \cos (\omega t)+\sin (\gamma) \sin (\omega t)) \\
\left.+\frac{1}{\sqrt{2}} I_{E_{0}}(\cos (\gamma) \sin (\omega t)-\sin (\gamma) \cos (\omega t))\right] \\
=2 \cos \left(\omega\left(\left[\left(I_{3} I_{S_{0}}\right) \cdot \vec{r}\right]\right)\right)\left[\frac{1}{\sqrt{2}} \cos (\omega t+\gamma)+\frac{1}{\sqrt{2}} I_{S_{0}} \sin (\omega t+\gamma)\right. \\
\left.+\frac{1}{\sqrt{2}} I_{B_{0}} \cos (\omega t-\gamma)+\frac{1}{\sqrt{2}} I_{E_{0}} \sin (\omega t-\gamma)\right] \\
=2 \cos \omega\left[\left(I_{3} I_{S}\right) \cdot \vec{r}\right]\left[\frac{1}{\sqrt{2}} \cos (\omega t+\gamma)+\frac{1}{\sqrt{2}} I_{S_{0}} \sin (\omega t+\gamma)\right. \\
\left.+\left(\frac{1}{\sqrt{2}} \cos (\omega t-\gamma)+\frac{1}{\sqrt{2}} I_{S_{0}} \sin (\omega t-\gamma)\right) I_{B_{0}}\right]
\end{aligned}
$$

The expression in the second square bracket is sum of operator of continuous rotation in plane $I_{S_{0}}$ with angular velocity $\omega$, angle is advanced by $\gamma$, and operator of continuous rotation in plane $I_{S_{0}}$ with angular velocity $\omega$, angle is retired by $\gamma$, with subsequent flip in plane $I_{S_{0}}$. The first one is lift of $|0\rangle$ in Dirac notations, the second one of $|1\rangle[5]$.

- Scattering element is $\cos (\gamma)+I_{B_{0}} \sin (\gamma)=\mathrm{e}^{I_{B_{0}} \gamma}$ with the result:

$$
\begin{aligned}
& \left(\cos (\gamma)+I_{B_{0}} \sin (\gamma)\right) \operatorname{SpSp}\left(\omega, \vec{r}, t, I_{S_{0}}, I_{B_{0}}, I_{E_{0}}\right)\left(\omega, \vec{r}, I_{S_{0}}, I_{B_{0}}, I_{E_{0}}\right) \\
& =2 \cos \left(\omega\left(\left[\left(I_{3} I_{S_{0}}\right) \cdot \vec{r}\right]\right)\right)\left[\cos (\omega t) \sin \left(\frac{\pi}{4}-\gamma\right)+I_{S_{0}} \sin (\omega t) \sin \left(\frac{\pi}{4}-\gamma\right)\right. \\
& \left.+I_{B_{0}} \cos (\omega t) \sin \left(\frac{\pi}{4}+\gamma\right)+I_{E_{0}} \sin (\omega t) \sin \left(\frac{\pi}{4}+\gamma\right)\right] \\
& =2 \cos \left(\omega\left(\left[\left(I_{3} I_{S_{0}}\right) \cdot \vec{r}\right]\right)\right)\left[\cos (\omega t) \sin \left(\frac{\pi}{4}-\gamma\right)+I_{S_{0}} \sin (\omega t) \sin \left(\frac{\pi}{4}-\gamma\right)\right. \\
& \left.+\left(\cos (\omega t) \sin \left(\frac{\pi}{4}+\gamma\right)+I_{S_{0}} \sin (\omega t) \sin \left(\frac{\pi}{4}+\gamma\right)\right) I_{B_{0}}\right]
\end{aligned}
$$

The variant with $I_{E_{0}}$ follows from similar calculation.

\section{Measurements by Wave Functions}

Let a $G_{3}^{+}$element $C_{0}+C_{1} I_{S_{0}}+C_{2} I_{B_{0}}+C_{3} I_{E_{0}}$, represented in bivector basis 
$\left\{I_{S_{0}}, I_{B_{0}}, I_{E_{0}}\right\}$, sits at point $\vec{r}$, and the sprefield interacts with it through measurement general formula implemented in geometric algebra terms as Hopf fibration:

$$
\begin{aligned}
& C_{0}+C_{1} B_{1}+C_{2} B_{2}+C_{3} B_{3} \stackrel{\alpha+\beta_{1} B_{1}+\beta_{2} B_{2}+\beta_{3} B_{3}}{\longrightarrow} C_{0} \\
& +\left(C_{1}\left[\left(\alpha^{2}+\beta_{1}^{2}\right)-\left(\beta_{2}^{2}+\beta_{3}^{2}\right)\right]+2 C_{2}\left(\beta_{1} \beta_{2}-\alpha \beta_{3}\right)+2 C_{3}\left(\alpha \beta_{2}+\beta_{1} \beta_{3}\right)\right) B_{1} \\
& +\left(2 C_{1}\left(\alpha \beta_{3}+\beta_{1} \beta_{2}\right)+C_{2}\left[\left(\alpha^{2}+\beta_{2}^{2}\right)-\left(\beta_{1}^{2}+\beta_{3}^{2}\right)\right]+2 C_{3}\left(\beta_{2} \beta_{3}-\alpha \beta_{1}\right)\right) B_{2} \\
& +\left(2 C_{1}\left(\beta_{1} \beta_{3}-\alpha \beta_{2}\right)+2 C_{2}\left(\alpha \beta_{1}+\beta_{2} \beta_{3}\right)+C_{3}\left[\left(\alpha^{2}+\beta_{3}^{2}\right)-\left(\beta_{1}^{2}+\beta_{2}^{2}\right)\right]\right) B_{3}
\end{aligned}
$$

The result in the current case is:

$$
\begin{aligned}
& C_{0}+C_{1} I_{S_{0}}+C_{2} I_{B_{0}}+C_{3} I_{E_{0}} \stackrel{\alpha+\beta_{1} I_{S_{0}}+\beta_{2} I_{B_{0}}+\beta_{3} I_{E_{0}}}{\longrightarrow} \\
& O\left(C_{0}, C_{1}, C_{2}, C_{3}, I_{S_{0}}, I_{B_{0}}, I_{E_{0}}, \omega, t, \vec{r}\right) \\
& =4\left(\cos \left(\omega\left[\left(I_{3} I_{S_{0}}\right) \cdot \vec{r}\right]\right)\right)^{2}\left[C_{0}+C_{3} I_{S_{0}}+\left(C_{1} \sin (2 \omega t)+C_{2} \cos (2 \omega t)\right) I_{B_{0}}\right. \\
& \left.\quad+\left(C_{2} \sin (2 \omega t)-C_{1} \cos (2 \omega t)\right) I_{E_{0}}\right]
\end{aligned}
$$

where

$$
\begin{aligned}
& \alpha=\sqrt{2} \cos \left(\omega\left(\left[\left(I_{3} I_{S_{0}}\right) \cdot \vec{r}\right]\right)\right) \cos (\omega t), \quad \beta_{1}=\sqrt{2} \cos \left(\omega\left(\left[\left(I_{3} I_{S_{0}}\right) \cdot \vec{r}\right]\right)\right) \sin (\omega t), \\
& \beta_{2}=\sqrt{2} \cos \left(\omega\left(\left[\left(I_{3} I_{S_{0}}\right) \cdot \vec{r}\right]\right)\right) \cos (\omega t), \quad \beta_{3}=\sqrt{2} \cos \left(\omega\left(\left[\left(I_{3} I_{S_{0}}\right) \cdot \vec{r}\right]\right)\right) \sin (\omega t)
\end{aligned}
$$

Geometrically that means that the measured element is rotated by $\frac{\pi}{2}$ in the $I_{B_{0}}$ plane, such that the $C_{3} I_{E_{0}}$ bivector component becomes lying in plane $I_{S_{0}}$ and remains unchanged, along with scalar component $C_{0}$. Two other bivector components became orthogonal to $I_{S_{0}}$ and continue rotating in $I_{S_{0}}$ with angular velocity $2 \omega t$.

Thus, we have what is called "collapse" in conventional quantum mechanics of the wave function, sprefield, at point $\vec{r}$, and the value of the collapse is "particle". Static $G_{3}^{+}$element is transformed into $G_{3}^{+}$element rotating with constant angular velocity in constant plane.

Quantum computers and quantum cryptography are maybe the first potential commercial applications of quantum physics. They can get their effective practical implementation only if formalized in terms of adequate logical and mathematical structure, and that has not fully achieved yet. Quantum mechanics in its existing formulation is a no-work-around obstacle for creating quantum computers. Huge efforts have been made in the area without successful results due to working with inappropriate mathematical tools. The core of quantum computing should be not in entanglement formally following from conventional quantum mechanics representation of many state systems as tensor products, which do not have clear geometrical or physical interpretation, of individual states.

The scheme suggested in the current text is based on manipulation and transferring of quantum states as operators acting on observables, both formulated in 
terms of geometrical algebra. In this way quantum computer appeared to be a kind of analog computer keeping and processing information by and on sets of objects possessing infinite number of degrees of freedom.

The very senseless wave/particle "dualism" cannot be seriously considered since the two are different things. Wave functions, thoroughly considered by P. Dirac as operators, under the name of "states", act on observables, producing "particles" if you prefer, through measurements.

Our states as elements of $G_{3}^{+}$are naturally mapped onto unit sphere $\mathbb{S}^{3}$. Two-state system is then just a couple of points on $\mathbb{S}^{3}$, say

$$
\begin{gathered}
\mathrm{e}^{I_{S_{1}} \varphi_{1}}=\alpha_{1}+I_{S_{1}} \beta_{1}=\alpha_{1}+\beta_{1} b_{1}^{1} B_{1}+\beta_{1} b_{1}^{2} B_{2}+\beta_{1} b_{1}^{3} B_{3} \\
\mathrm{e}^{I_{S_{2}} \varphi_{2}}=\alpha_{2}+I_{S_{2}} \beta_{2}=\alpha_{2}+\beta_{2} b_{2}^{1} B_{1}+\beta_{2} b_{2}^{2} B_{2}+\beta_{2} b_{2}^{3} B_{3}
\end{gathered}
$$

where

$$
\begin{gathered}
\alpha_{1}^{2}+\beta_{1}^{2}\left(b_{1}^{1^{2}}+b_{1}^{2^{2}}+b_{1}^{3^{2}}\right)=\alpha_{1}^{2}+\beta_{1}^{2}=1 \text { and } \\
\alpha_{2}^{2}+\beta_{2}^{2}\left(b_{2}^{1^{2}}+b_{2}^{2^{2}}+b_{2}^{3^{2}}\right)=\alpha_{2}^{2}+\beta_{2}^{2}=1
\end{gathered}
$$

in some bivector basis $B_{1} B_{2} B_{3}=1, B_{1} B_{2}=-B_{3}, B_{1} B_{3}=B_{2}, B_{2} B_{3}=-B_{1}$.

Then it follows that two states of an arbitrary two-state system are, in any case, connected by the Clifford translation ${ }^{2}$ :

$$
\mathrm{e}^{I_{S_{2}} \varphi_{2}}=\left(\mathrm{e}^{I_{S_{2}} \varphi_{2}} \mathrm{e}^{-I_{S_{1}} \varphi_{1}}\right) \mathrm{e}^{I_{S_{1}} \varphi_{1}} \equiv C l\left(S_{2}, \varphi_{2}, S_{1}, \varphi_{1}\right) \mathrm{e}^{I_{S_{1}} \varphi_{1}}
$$

The product of exponents $\mathrm{e}^{I_{S_{2}} \varphi_{2}} \mathrm{e}^{-I_{S_{1}} \varphi_{1}}$ is trivial in the case $S_{1}=S_{2} \equiv S$ (the case of unspecified imaginary unit in conventional quantum mechanics) $\mathrm{e}^{I_{S_{2}} \varphi_{2}} \mathrm{e}^{-I_{S_{1}} \varphi_{1}}=\mathrm{e}^{I_{S}\left(\varphi_{2}-\varphi_{1}\right)}$. Though in general case we also know more complicated result:

$$
\begin{aligned}
& C l\left(S_{2}, \varphi_{2}, S_{1}, \varphi_{1}\right) \equiv \mathrm{e}^{I_{S_{2}} \varphi_{2}} \mathrm{e}^{-I_{S_{1}} \varphi_{1}} \\
& =\cos \left(\varphi_{1}\right) \cos \left(\varphi_{2}\right)+\left(s_{1} \cdot S_{2}\right) \sin \left(\varphi_{1}\right) \sin \left(\varphi_{2}\right)+I_{3} S_{2} \cos \left(\varphi_{1}\right) \sin \left(\varphi_{2}\right) \\
& \quad+I_{3} s_{1} \cos \left(\varphi_{2}\right) \sin \left(\varphi_{1}\right)+I_{3}\left(s_{2} \times s_{1}\right) \sin \left(\varphi_{1}\right) \sin \left(\varphi_{2}\right)
\end{aligned}
$$

where $S_{1}$ and $S_{2}$ are vectors dual to planes $S_{1}$ and $S_{2}$ relative to orientation of $I_{3}$.

From knowing Clifford translation connecting any two states as points on $\mathbb{S}^{3}$ it follows that the result of measurement of any observable $C$ by the state $\mathrm{e}^{I_{S_{1}} \varphi_{1}}$, for example $\mathrm{e}^{I_{S_{1}} \varphi_{1}} C \mathrm{e}^{-I_{S_{1}} \varphi_{1}} \equiv C\left(S_{1}, \varphi_{1}\right)$, immediately gives the result of (not made) measurement by $\mathrm{e}^{I_{S_{2}} \varphi_{2}}$ :

$$
\begin{aligned}
\mathrm{e}^{I_{S_{2}} \varphi_{2}} C \mathrm{e}^{-I_{S_{2}} \varphi_{2}} & =\mathrm{e}^{I_{S_{2}} \varphi_{2}} \mathrm{e}^{-I_{S_{1}} \varphi_{1}} \mathrm{e}^{I_{S_{1}} \varphi_{1}} C \mathrm{e}^{-I_{S_{1}} \varphi_{1}} \mathrm{e}^{I_{S_{1}} \varphi_{1}} \mathrm{e}^{-I_{S_{2}} \varphi_{2}} \\
& =\mathrm{e}^{I_{S_{2}} \varphi_{2}} \mathrm{e}^{-I_{S_{1}} \varphi_{1}} C\left(S_{1}, \varphi_{1}\right) \mathrm{e}^{I_{S_{1}} \varphi_{1}} \mathrm{e}^{-I_{S_{2}} \varphi_{2}} \\
& =C l\left(S_{2}, \varphi_{2}, S_{1}, \varphi_{1}\right) C\left(S_{1}, \varphi_{1}\right) \overline{C l\left(S_{2}, \varphi_{2}, S_{1}, \varphi_{1}\right)}
\end{aligned}
$$

This is geometrically clear and unambiguous explanation of strict connectivity ${ }^{2}$ It is universally possible due to the hedgehog theorem.

${ }^{3}$ Difference in exponent signs from usual measurement definition is made just for some convenience. It means that the angle has opposite sign or can be thought that the bivector plane was flipped. 
of the results of measurements instead of quite absurd "entanglement" in conventional quantum mechanics.

The received formula:

$$
\begin{aligned}
& 4\left(\cos \left(\omega\left[\left(I_{3} I_{S_{0}}\right) \cdot \vec{r}\right]\right)\right)^{2}\left[C_{0}+C_{3} I_{S_{0}}+\left(C_{1} \sin (2 \omega t)+C_{2} \cos (2 \omega t)\right) I_{B_{0}}\right. \\
& \left.+\left(C_{2} \sin (2 \omega t)-C_{1} \cos (2 \omega t)\right) I_{E_{0}}\right]
\end{aligned}
$$

shows that only component of the result of measurement lying in plane $I_{S_{0}}$ does not depend on the value of time parameter. We know that any two observables can be connected through Clifford translation. If we are reasonably concerned only in the $I_{S_{0}}$ component of the result of measurement then with placing another observable value $C_{3}^{\text {new }}$ the latter can be written, in assumption that the $I_{S_{0}}$ old observable component is not zero, as:

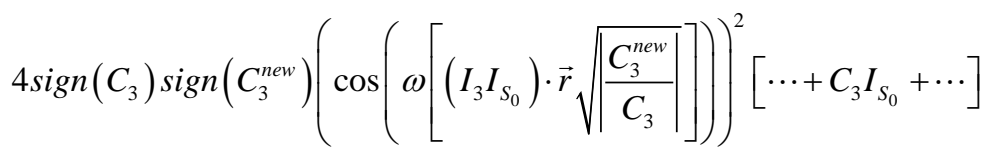

Thus, all the $I_{S_{0}}$ components of any observable do simultaneously exist whilst we only made measurement of one observable. All the other observables values are stored at distances $\vec{r} \sqrt{\left|\frac{C_{3}^{\text {new }}}{C_{3}}\right|}$.

Consider some cases when the sprefield is scattered before measuring.

When sprefield is scattered on element $\cos (\gamma)+I_{S_{0}} \sin (\gamma)=\mathrm{e}^{I_{S_{0}} \gamma}$ we have:

$\alpha=\sqrt{2} \cos \left(\omega\left(\left[\left(I_{3} I_{S_{0}}\right) \cdot \vec{r}\right]\right)\right) \cos (\omega t+\gamma), \quad \beta_{1}=\sqrt{2} \cos \left(\omega\left(\left[\left(I_{3} I_{S_{0}}\right) \cdot \vec{r}\right]\right)\right) \sin (\omega t+\gamma)$, $\beta_{2}=\sqrt{2} \cos \left(\omega\left(\left[\left(I_{3} I_{S_{0}}\right) \cdot \vec{r}\right]\right)\right) \cos (\omega t-\gamma), \quad \beta_{3}=\sqrt{2} \cos \left(\omega\left(\left[\left(I_{3} I_{S_{0}}\right) \cdot \vec{r}\right]\right)\right) \sin (\omega t-\gamma)$

and

$$
\begin{aligned}
& C_{0}+C_{1} I_{S_{0}}+C_{2} I_{B_{0}}+C_{3} I_{E_{0}} \stackrel{\alpha+\beta_{1} I_{S_{0}}+\beta_{2} I_{B_{0}}+\beta_{3} I_{E_{0}}}{\longrightarrow} \\
& O\left(C_{0}, C_{1}, C_{2}, C_{3}, I_{S_{0}}, I_{B_{0}}, I_{E_{0}}, \omega, t, \vec{r}\right) \\
& =4\left(\cos \left(\omega\left[\left(I_{3} I_{S_{0}}\right) \cdot \vec{r}\right]\right)\right)^{2}\left[C_{0}+\left(2 C_{2} \sin (2 \gamma)+2 C_{3} \cos (2 \gamma)\right) I_{S_{0}}\right. \\
& \quad+\left(2 C_{1} \sin (2 \omega t)+2 C_{2} \cos (\omega t) \cos (\gamma)-2 C_{3} \sin (\omega t) \cos (\gamma)\right) I_{B_{0}} \\
& \left.\quad+\left(-C_{1} \cos (2 \omega t)+2 C_{2} \sin (\omega t) \cos (\gamma)-2 C_{3} \sin (\omega t) \sin (\gamma)\right) I_{E_{0}}\right]
\end{aligned}
$$

Again, we have constant value in plane $I_{S_{0}}$ and rotation of two other bivector components orthogonal to it.

In the case of scattering element $\cos (\gamma)+I_{B_{0}} \sin (\gamma)=\mathrm{e}^{I_{B_{0}} \gamma}$ we have

$$
\begin{aligned}
& \alpha=\sqrt{2} \cos \left(\omega\left(\left[\left(I_{3} I_{S_{0}}\right) \cdot \vec{r}\right]\right)\right) \cos (\omega t) \sin \left(\frac{\pi}{4}-\gamma\right), \\
& \beta_{1}=\sqrt{2} \cos \left(\omega\left(\left[\left(I_{3} I_{S_{0}}\right) \cdot \vec{r}\right]\right)\right) \sin (\omega t) \sin \left(\frac{\pi}{4}-\gamma\right),
\end{aligned}
$$




$$
\begin{aligned}
& \beta_{1}=\sqrt{2} \cos \left(\omega\left(\left[\left(I_{3} I_{S_{0}}\right) \cdot \vec{r}\right]\right)\right) \sin (\omega t) \sin \left(\frac{\pi}{4}-\gamma\right) \\
& \beta_{3}=\sqrt{2} \cos \left(\omega\left(\left[\left(I_{3} I_{S_{0}}\right) \cdot \vec{r}\right]\right)\right) \sin (\omega t) \sin \left(\frac{\pi}{4}+\gamma\right)
\end{aligned}
$$

and

$$
\begin{aligned}
& C_{0}+C_{1} I_{S_{0}}+C_{2} I_{B_{0}}+C_{3} I_{E_{0}} \stackrel{\alpha+\beta_{1} I_{S_{0}}+\beta_{2} I_{B_{0}}+\beta_{3} I_{E_{0}}}{\longrightarrow} \\
& O\left(C_{0}, C_{1}, C_{2}, C_{3}, I_{S_{0}}, I_{B_{0}}, I_{E_{0}}, \omega, t, \vec{r}\right) \\
& =4\left(\cos \left(\omega\left[\left(I_{3} I_{S_{0}}\right) \cdot \vec{r}\right]\right)\right)^{2}\left[C_{0}+\left(-C_{1} \sin (2 \gamma)+2 C_{3} \cos (2 \gamma)\right) I_{S_{0}}\right. \\
& +\left(C_{1} \sin (2 \omega t) \cos (2 \gamma)-C_{2} \cos (2 \omega t) \sin (2 \gamma)-2 C_{3} \sin (2 \omega t) \sin (2 \gamma)\right) I_{B_{0}} \\
& \left.+\left(-2 C_{1} \cos (2 \omega t) \sin (2 \gamma)+2 C_{2} \sin (2 \omega t)-C_{3} \cos (2 \omega t) \sin (2 \gamma)\right) I_{E_{0}}\right]
\end{aligned}
$$

\section{Conclusions}

Wave function when implemented as special solution of the Maxwell equation in geometric algebra terms is spread through the whole three-dimensional space and time parameter values. If scattered by another $G_{3}^{+}$element the result is simultaneously redefined everywhere and for all values of $t$. We particularly have changing of wave function backward in time. That is obvious demonstration that the suggested theory allows indefinite event casual order. In that way the very notion of the concept of cause and effect disappears, thus we might not perceive time.

In measurements, through applying wave function to a static $G_{3}^{+}$element, the result is new $G_{3}^{+}$element rotating in not changing plane with constant angular velocity- "particle", that is "collapse" of wave function.

\section{Conflicts of Interest}

The author declares no conflicts of interest regarding the publication of this paper.

\section{References}

[1] Soiguine, A. (2015) Geometric Phase in Geometric Algebra Qubit Formalism. Lambert Academic Publishing, Saarbrucken.

[2] Soiguine, A. (2018) State/Observable Interactions Using Basic Geometric Algebra Solutions of the Maxwell Equation. arXiv:1807.08603.

[3] Soiguine, A. (1996) Complex Conjugation-Relative to What? In: Abłamowicz, R., Parra, J.M. and Lounesto, P., Eds., Clifford Algebras with Numeric and Symbolic Computations, Birkhauser, Cambridge, MA, 285-294. https://arxiv.org/abs/1607.03413

[4] Soiguine, A. (2016) Derivation of the Schrodinger Equation in Geometric Algebra. https://www.researchgate.net/publication/301593591_Derivation_of_the_Schroding er_equation_in_geometric_algebra

[5] Soiguine, A. (2016) Anyons in Three Dimensions with Geometric Algebra. https://arxiv.org/abs/1607.03413 